\title{
NUEVOS MÉTODOS PARA LA DETECCIÓN DE OBSTÁCULOS INESPERADOS DURANTE LA MARCHA NORMAL A TRAVÉS DE SEÑALES EEG
}

\author{
María Elvira, Eduardo Iáñez, Vicente Quiles, Mario Ortiz, José M.Azorín \\ Brain-Machine Interface Systems Lab, Miguel Hernández University of Elche \\ Avda. de la Universidad s/n, Ed. Innova, 03202, Elche, Spain \\ maria.elvira01@goumh.umh.es, eianez@umh.es, vquiles@umh.es, mortiz@umh.es, jm.azorin@umh.es,
}

\begin{abstract}
Resumen
El objetivo del presente trabajo es evaluar nuevos métodos que permitan detectar la aparición de un obstáculo inesperado durante la marcha normal a partir de señales electroencefalográficas (EEG), reduciendo la tasa de falsos positivos obtenida en estudios previos. De esta forma, se podría detener en caso de emergencia un exoesqueleto destinado tanto a tareas de rehabilitación como de asistencia a personas con alguna discapacidad motora. Se pretende así ir aproximándonos a una implementación de este en la vida real, consiguiendo una mayor interacción e implicación del sujeto con el sistema. Se ha conseguido una mejora en los resultados obtenidos respecto a un estudio previo, obteniendo un $75.0 \%$ de acierto y 4.5 falsos positivos por minuto $(\mathrm{FP} / \mathrm{min})$.
\end{abstract}

Palabras clave: obstáculo, EEG, BMI, exoesqueleto.

\section{INTRODUCCIÓN}

La utilización de exoesqueletos durante las terapias de rehabilitación enfocadas a pacientes con alguna discapacidad física presenta grandes beneficios para la recuperación de la movilidad de la zona perdida. La tasa de pacientes afectados por una discapacidad motora está aumentando debido al envejecimiento de la población y al creciente número de enfermedades crónicas, situándose en un $15 \%$ de la población mundial, según la OMS. Esto explica el creciente interés de la comunidad científica por el desarrollo de interfaces BMI (Brain-Machine Interfaces) que permitan una mayor involucración del paciente en la rehabilitación y, consecuentemente, una mejor recuperación [12].

De hecho, se ha demostrado que el tratamiento de rehabilitación en los primeros 6 meses tras sufrir un accidente cerebrovascular (ACV) supone el camino más efectivo para la recuperación de la movilidad perdida, debido a la plasticidad del sistema nervioso durante este tiempo [8]. Es en esta etapa, sin embargo, donde la rehabilitación tradicional supone grandes dificultades para muchos pacientes por su incapacidad para realizar los movimientos pertinentes o por el excesivo esfuerzo físico que les supone la terapia.

En este ámbito varios estudios han llevado a cabo un análisis de la marcha detectando, a través de señales electroencefalográficas (EEG), diferentes intenciones del usuario, como iniciar la marcha, detenerse, o realizar un cambio de dirección voluntario [1]. Asimismo, se ha estudiado la detección de la intención de parar por parte del usuario ante la aparición de un obstáculo inesperado [9] [10]. En este último caso, ha sido posible el reconocimiento de dicha señal antes de que el sujeto se detenga. Esto permitiría enviar una orden de parada a un exoesqueleto en caso de producirse una emergencia, posibilitando la implementación de este en la vida real de manera totalmente confiable, útil y segura [5].

Siguiendo esta línea, el objetivo principal del presente trabajo es la exploración de nuevos métodos de extracción de características y clasificación para obtener resultados más favorables en la detección de obstáculos inesperados por parte del sistema BMI. Utilizando una BMI no invasiva, se procederá a reconocer la reacción producida en el cerebro ante la aparición repentina de un obstáculo visual. Por lo tanto, se espera encontrar principalmente la actividad cerebral tanto en la zona occipital, debido al estímulo visual, como en la zona motora, puesto que hay una intención del usuario de mandar una señal de detención al sistema muscular. Se consigue, mediante el análisis de las señales EEG, una mayor implicación e interacción del usuario que la que se obtendría con sistemas basados en cámaras colocados en el exoesqueleto [9].

\section{MATERIAL Y MÉTODOS}

A continuación, se describe el equipo software y hardware utilizado en las pruebas, así como el protocolo experimental mantenido. Además, se detalla el método de preprocesamiento para el 
filtrado de la señal EEG, los diferentes métodos de extracción de características propuestos y el clasificador seleccionado. Por último, se describe la estrategia seguida para el análisis pseudo-online.

\subsection{CONFIGURACIÓN EXPERIMENTAL}

Para el registro de las señales EEG se ha utilizado el equipo g.USBamp de la marca g.Tec, formado por dos amplificadores de 16 canales de entrada cada uno, que permiten registrar la señal de un total de 32 electrodos con una frecuencia de muestreo de 1200 $\mathrm{Hz}$. Ambos equipos han sido sincronizados mediante el dispositivo g.INTERsync.

La disposición de los electrodos sigue el Sistema Internacional 10/10, manteniendo el siguiente orden: Fz, FC5, FC1, FCz, FC2, FC6, C3, Cz, C4, CP5, CP1, CP2, CP6, P3, Pz, P4, PO7, PO3, PO4, PO8, FC3, FC4, C5, C1, C2, C6, CP3, CPZ, CP4, P1, P2 y POz. El lóbulo de la oreja derecha ha sido empleado como referencia y el electrodo situado en la posición $\mathrm{AFz}$ como tierra. El análisis y procesado de los datos se ha llevado a cabo mediante el software Matlab.

Por otra parte, el equipo Tech MCS V3 ha sido el seleccionado para llevar a cabo un seguimiento del movimiento de la persona, permitiendo conocer los instantes en la que ésta se encuentra andando o parada. Su utilización queda justificada por la necesidad de detectar un cambio en la señal EEG como reacción al estímulo anterior a la parada de la persona. Concretamente, se han utilizado 7 unidades de medición inercial (IMUs) dispuestas de la siguiente manera: lumbar, muslo derecho/ izquierdo, espinilla derecha/izquierda, pie derecho/ izquierdo. Para cada sensor se han tenido en cuenta 3 de los 19 parámetros obtenidos individualmente: 'Acc X', 'Acc Y' y 'Acc Z', a partir de los cuales, y mediante el empleo de una Transformada Wavelet Continua (CWT), se ha conseguido detectar las diferentes paradas [7].

Como equipo adicional se incluye una máquina de correr Performance 750 de la marca Pro-form así como una línea láser proyectada en la parte delantera de la cinta de correr, con una longitud de onda de 635 $\mathrm{nm}$ (color rojo) y una potencia de salida de $3 \mathrm{~mW}$. Este láser ha sido controlado y sincronizado con el registro de datos en Matlab.

En la figura 1 se puede observar una fotografía de todo el montaje experimental.

\subsection{PROCEDIMIENTO EXPERIMENTAL}

Los registros se han llevado a cabo en 4 sujetos sanos, sin ningún desorden neurológico, con edades

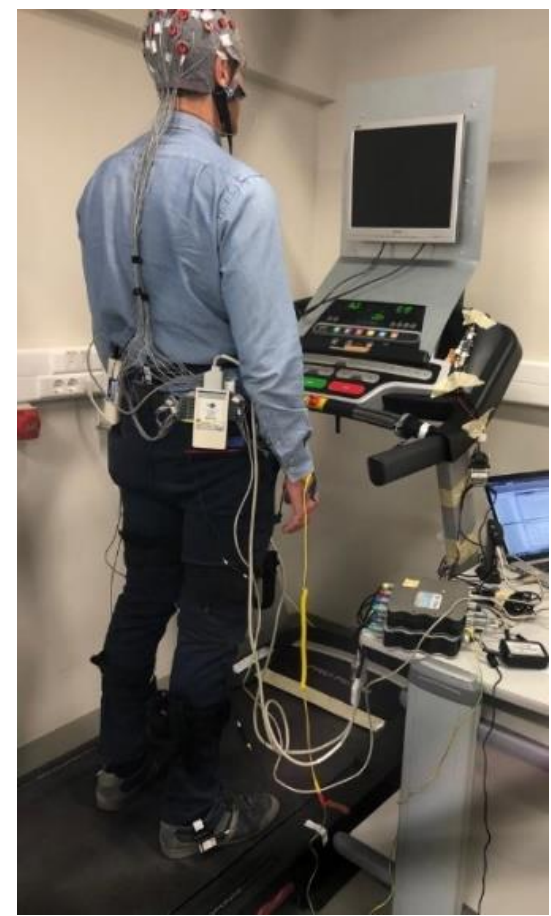

Figura 1: Montaje experimental de la prueba.

comprendidas entre 21 y 26 años, tres de ellos diestros y uno zurdo, y sin ningún problema de visión. Cada uno de ellos ha realizado 10 registros de reacción frente a la aparición de un láser, a excepción de $\mathrm{S} 1$ que únicamente realizó 5. Cada registro tiene una duración de 120 segundos, durante los cuales se proyecta de manera variable la línea láser con una duración de encendido de 1 segundo y un tiempo entre dos estímulos sucesivos aleatorio comprendido entre 6 y 9 segundos. El número total de láseres por sesión varía entre 12 y 14 .

La prueba inicia con la cinta de correr parada, instante en el cual se procede al calibrado de las IMUs; a continuación, se enciende la máquina y el sujeto comienza a andar a un ritmo prefijado constante de $2 \mathrm{~km} / \mathrm{h}$ y una inclinación de 0 grados. Cuando la persona se encuentra andando de manera libre ( $\sin$ cogerse a la máquina) y estable, se procede a iniciar el registro.

\subsection{PREPROCESAMIENTO}

Para el preprocesamiento de la señal, en primer lugar, se han descartado para el estudio algunos electrodos afectados por artefactos de movimiento debido a que están localizados en la periferia de la cabeza [9]- FC5, FC6, CP5, CP6, PO3, PO4, PO7, PO8, C5 y C6- Posteriormente se ha aplicado un filtro paso-banda de 0.4 a $3 \mathrm{~Hz}$ según lo demostrado en [10]. 
Por último, se ha aplicado un filtro CAR (en inglés, Common Average Reference filter), para suavizar las contribuciones de la actividad cerebral basal. Las señales filtradas con una desviación estándar de más de $40 \mu \mathrm{V}$ han sido eliminadas [9].

\subsection{PROCESAMIENTO Y EXTRACCIÓN DE CARACTERÍSTICAS}

A continuación, se explica el procedimiento elegido para la obtención de los modelos, el cual podemos estructurar en tres pasos- selección personalizada de electrodos, selección personalizada de ventanas temporales y extracción de características- y el clasificador empleado.

\subsubsection{Selección personalizada de electrodos}

En el cerebro encontramos cuatro lóbulos cerebralesestos son frontal, temporal, parietal y occipital [3]. Dado que la señal EEG que estamos buscando es respuesta a un estímulo visual inesperado, que produce una intención del usuario de parar, las zonas objeto de estudio serán el área motora, área sensitiva y área occipital, localizadas respectivamente en el lóbulo frontal, parietal y occipital. Es por ello por lo que nos focalizaremos en los electrodos dispuestos en estas regiones- $\mathrm{Fz}, \mathrm{FCz}, \mathrm{Cz}, \mathrm{C} 1, \mathrm{C} 2, \mathrm{CP} 1, \mathrm{CP} 2$, CP3, CP4, CPZ, P1, P2, P3, P4 y POz. Ahora bien, para cada sujeto se ha realizado una preselección de aquellos electrodos en los que se aprecia visualmente un mayor cambio cuando aparece el láser. Con este subconjunto de electrodos elegidos individualmente se ha procedido a la suma de la señal de los mismos. A partir de esta suma, se ha realizado la obtención de ventanas de láser (clase 1) y no láser (clase 2) y la extracción de los respectivos vectores de características para la obtención del modelo.

A continuación, se muestra la comparación entre la señal obtenida eligiendo todos los electrodos de la zona motora y occipital (figura 2), y la obtenida incluyendo sólo los del subconjunto elegido individualmente (figura 3) para el sujeto S2. En ambas figuras se muestra la señal recogida cuando el usuario está caminando normal, hasta el instante de aparición de láser (representado en línea roja). A partir de dicha línea roja se muestran los dos segundos posteriores en los que se registra la reacción por parte del usuario. En azul aparecen superpuestas distintas activaciones del láser; en negro, el promedio de todas ellas. Se observa cómo, en el segundo caso, la señal es más homogénea y únicamente hay cambio significativo tras el láser.

La tabla 1 recoge el subconjunto de electrodos elegidos para cada usuario cuya señal se sumará y se procesará según lo indicado en los siguientes apartados.

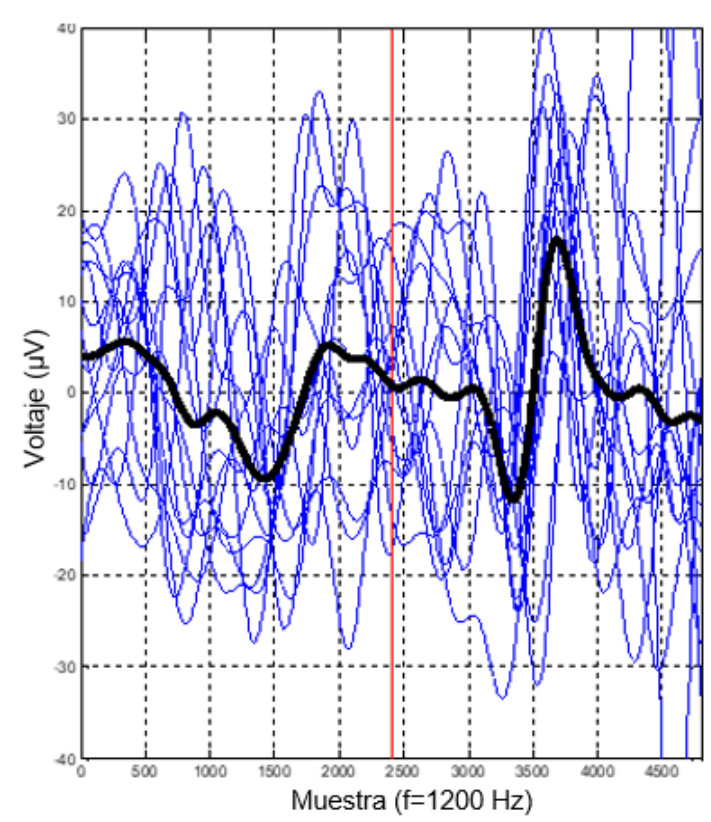

Figura 2: Suma de todos los electrodos de la zona occipital y motora en S2.

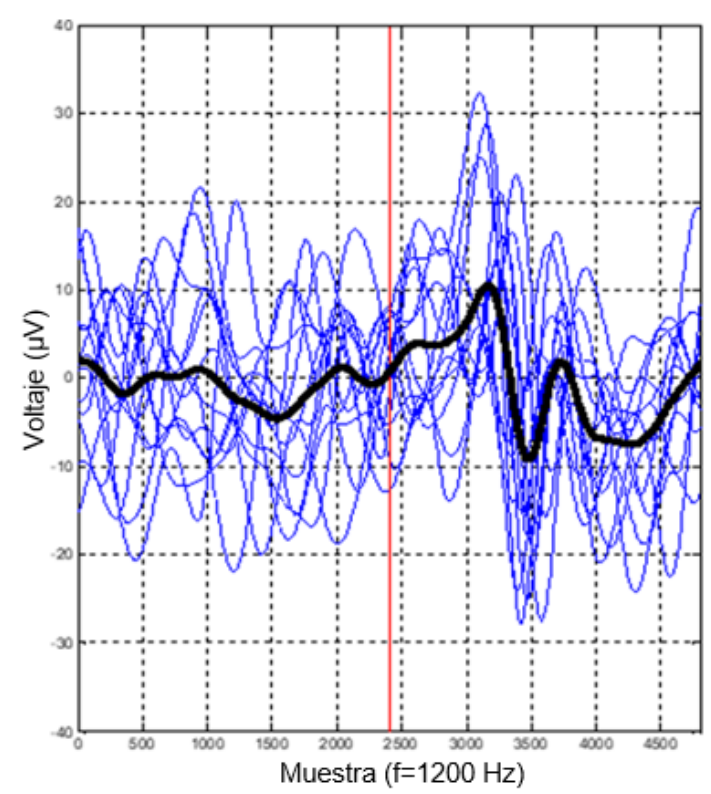

Figura 3: Suma del subconjunto de electrodos seleccionados $\mathrm{S} 2$.

Tabla 1. Selección de electrodos para cada sujeto

\begin{tabular}{|c|c|}
\hline Sujeto & Electrodos \\
\hline S1 & $\begin{array}{r}\text { FZ, FCZ, CZ, CP1, CP2, P3, C1, } \\
\text { CPZ, P1, P2, CP3, CP4, POZ }\end{array}$ \\
\hline S2 & $\begin{array}{r}\text { FZ, FCZ, CZ, CP1, CP2, P3, C1, } \\
\text { CPZ, P1, P2, CP3, CP4 }\end{array}$ \\
\hline S3 & $\begin{array}{r}\text { FZ, FCZ, CZ, CP1, CP2, P3, P4, C1, } \\
\text { P1, P2, CP3, CP4, POZ }\end{array}$ \\
\hline S4 & FZ, CZ, P3, POZ \\
\hline
\end{tabular}




\subsubsection{Selección individualizada de ventanas de clase 1 (láser) para la obtención del modelo}

De la literatura sabemos que los ERP (Event-Related Potentials) son variaciones en la amplitud de las señales EEG producidas en el córtex cerebral por la suma de un gran número de potenciales de acción generados por estímulos cognitivos, afectivos o sensitivos [11]. En un estudio previo se determinó que, concretamente, la reacción ante un estímulo visual produce una deflexión positiva en la señal EEG unos $700 \mathrm{~ms}$ antes de la reacción, y una deflexión negativa $400 \mathrm{~ms}$ antes [10].

Sin embargo, respecto a estos tiempos, hay que tener en cuenta dos aspectos. En primer lugar, presentan cierto grado de variabilidad entre individuos; por otro lado, el tiempo de reacción de cada usuario puede variar ampliamente. Estas dos afirmaciones nos hicieron concluir que para escoger las ventanas temporales con las que crear el modelo debíamos seleccionar individualmente cuando se producía, en promedio, la mayor variación en la amplitud de la señal EEG.

Así pues, nuestro modelo ha sido obtenido de la siguiente manera. Dado que queremos distinguir entre dos clases distintas correspondientes al instante de aparición de láser- en adelante, clase 1- y a los momentos de no aparición del obstáculo- clase 2hemos extraído ventanas con una duración temporal de $500 \mathrm{~ms}$ correspondientes a ambas clases. La ventana de clase 2 ha sido escogida en todos los casos a partir de la señal comprendida entre 2 y 1.5 segundos antes de la aparición del láser. Sin embargo, la ventana correspondiente a la clase 1 no ha sido escogida siempre en el mismo instante, sino que hemos realizado un primer registro, a partir del cual se ha obtenido la señal promedio de todos los láseres (como la mostrada en la figura 3) y se ha detectado el instante en el que se produce el máximo de dicho promedio, localizándose en uno de los usuarios 700 muestras después del instante de aparición del láser (0.5833 s), en otro tan sólo 76 muestras después $(0.0633 \mathrm{~s})$ y en el resto de casos entre las muestras 200 y 300 posteriores al obstáculo. De esta manera, hemos conseguido captar una zona de mayor diferencia entre la clase 1 (láser) y la clase 2 (relax).

En la figura 4 aparece representada para el usuario S1 la superposición de la suma de todos los electrodos en el instante del láser. Si comparamos esta imagen con la figura 3, donde aparece la misma representación, pero para otro sujeto, podemos apreciar la diferencia de periodo de tiempo en el que hay un cambio significativo en la señal EEG. En el caso de la figura 4 se produce justo después del láser,

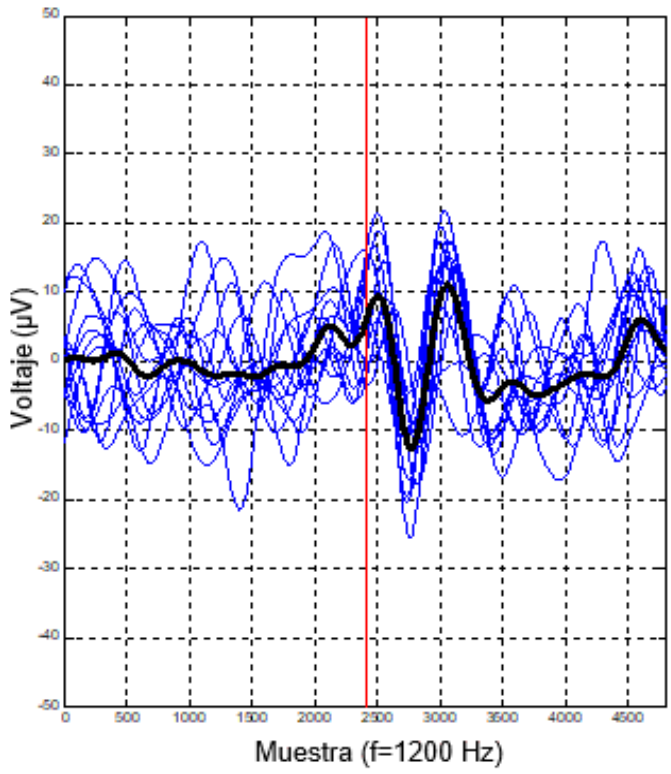

Figura 4. Superposición de la señal suma de la selección de electrodos en todos los instantes de láser para el usuario $\mathrm{S} 1$

mientras que en la figura 3 ocurre unas 700 muestras después.

\subsubsection{Extracción de características aplicada a cada ventana de análisis.}

Para esta etapa, se han probado tres vectores de características distintos aplicados tanto a las ventanas etiquetadas como clase 1 (láser) como a las de clase 2 (no láser). En todos ellos, los parámetros han sido extraídos a partir de la suma de la señal de los electrodos elegidos para cada usuario, a la cual denominaremos $f 1$, ya que al considerar todas estas contribuciones en conjunto se obtiene una variación más característica en los momentos de reacción.

A partir de esta señal $f 1$, se han extraído los vectores de características:

> Caso 1: se obtiene un vector de 5 características, feat (1: 5):, las cuales son:

1. Máximo de la correlación cruzada normalizada entre ambas señales

$$
f e a t(1)=\max (f 1 \star g 1)_{i}
$$

Siendo $f 1$ la suma para cada ventana de la señal de los electrodos elegidos individualmente y $g l$ la media de todas las fl. 
2. Amplitud de la suma acumulada del valor absoluto de $f 1, \operatorname{sum}(f 1)$ a lo largo de una ventana.

feat $(2)=\max (\operatorname{sum}(f 1))-\min (\operatorname{sum}(f 1))$

3. feat (3:5): tercer, cuarto y quinto término del polinomio de orden 5 , según lo indicado en [9], que permite el mejor ajuste por mínimos cuadrados a la señal $f 1$ (se descarta el resto de términos por una diferencia insignificante entre ambas clases).

> Caso 2: se ha utilizado igualmente un vector de 5 características, las cuales son:

1. Área comprendida bajo la curva del valor absoluto de la derivada de $f 1$.

$$
f e a t(1)=\int a b s\left(f 1^{\prime}\right)
$$

2. Varianza de la derivada de $f 1$.

$$
f e a t(2)=\operatorname{var}\left(f 1^{\prime}\right)
$$

3. Amplitud de la derivada de $f 1$.

$$
\text { feat }(3)=\max \left(f 1^{\prime}\right)-\min \left(f 1^{\prime}\right)
$$

4. Amplitud de la suma acumulada de la señal valor absoluto de $f 1$ a lo largo de una ventana (ídem que caso 1).

5. Máximo de la correlación cruzada entre $g 1$, y $f l$ en cada caso particular (ídem que caso 1.1).

$>$ Caso 3: vector de 4 características, idéntico al caso 2, a excepción de que se ha eliminado el coeficiente de correlación.

\subsection{CLASIFICADOR}

De acuerdo con lo indicado en [9], el clasificador escogido para la distinción entre las 2 clases ha sido un análisis discriminante lineal (LDA), el cual transforma un conjunto de datos d-dimensional en un subespacio $\mathrm{k}$-dimensional (con $\mathrm{k}<\mathrm{d}$ ), maximizando la distancia entre las medias de cada clase y, simultáneamente, minimizando sus dispersiones [13].

La función de discriminación es la siguiente:

$$
\begin{gathered}
h_{\beta}(x)=\left\{\begin{array}{l}
\left(\beta^{T} \cdot x+\beta_{o}\right) \geq 0 \rightarrow 1 \\
\left(\beta^{T} \cdot x+\beta_{o}\right)<0 \rightarrow 2
\end{array}\right. \\
\beta=\Sigma^{-1}\left(\mu_{1}-\mu_{2}\right)
\end{gathered}
$$

$$
\beta_{o}=-\beta^{T} \cdot\left(\frac{\mu_{1}+\mu_{2}}{2}\right)+\ln \left(\frac{\pi_{1}}{\pi_{2}}\right)
$$

Donde $\beta$ es el vector de parámetros de clasificación, $\beta_{o}$ es el término de sesgo, $\sum$ es la matriz de covarianza agrupada, $\mu_{1}$ y $\mu_{2}$ son los vectores medios de clase 1 y clase 2 , y $\pi_{1}$ y $\pi_{2}$ sus probabilidades a priori [2]. Estas probabilidades se han determinado en 4 para la clase 2 frente a 1 para la clase 1 respectivamente, al ser mucho más abundantes los casos de relax que de aparición del obstáculo.

\subsection{CLASIFICACIÓN PSEUDO-ONLINE}

Para la realización de la clasificación pseudo-online se ha procedido de la siguiente manera: todos los registros a excepción del último han sido utilizados para la creación del modelo, siendo este último el que ha sido analizado en pseudo-online. Para la obtención del modelo se ha realizado la selección de ventanas y extracción de características siguiendo lo indicado en 2.4.2 y 2.4.3. A continuación, tras aplicarle el preprocesamiento indicado en 2.3 , se ha analizado en pseudo-online el último fichero. Para ello se han ido estudiando ventanas temporales de $500 \mathrm{~ms}$ de longitud y solapadas $400 \mathrm{~ms}$, extrayendo el vector de características correspondiente en función del caso aplicado (véase sección 2.4.3) y clasificándolas como clase 1 o 2 .

Para el caso 3 de extracción de características mencionado en 2.4 .3 se ha añadido una segunda clasificación basada en la superación de un umbral por parte de los máximos de la correlación cruzada normalizada de cada electrodo, basada en lo establecido en [6], la cual nos ha servido para reforzar los verdaderos positivos y disminuir los falso positivos que se producen. Esta norma es la siguiente:

Si el clasificador obtiene como salida clase 1 y además se cumple que:

$$
\max (f 1 \star g 1)_{i} \geq 0.5
$$

se considera como clase 1 dicha ventana.

Si el clasificador obtiene como salida clase 2 , se hace una segunda validación, en la que si se cumple igualmente (9) y, además, se tiene que, para la señal individual de cada uno de los electrodos:

$$
\max (f i 1 \star h 1)_{i} \geq 0.5
$$

siendo $f i 1$ la señal de un electrodo individual en dicha ventana temporal y $h 1$ la señal promedio de 
ese mismo electrodo, entonces la ventana se clasifica igualmente como clase 1 .

En el resto de casos se considerará clase 2 (no láser).

Debido al solapamiento de ventanas mantenido, hay información redundante que puede provocar la detección de varias ventanas seguidas como láser cuando se ha producido únicamente una [4]. Como solución, se han calculado los resultados para distintos valores de $\mathrm{K}$, esto es, número de veces seguidas que deben clasificarse las ventanas como clase 1 para considerarse correcta la detección [9]. Esto se ha aplicado a todos los casos de extracción de características explicados en 2.4.3.

\section{RESULTADOS Y DISCUSIÓN}

En la tabla 2 quedan recogidos los resultados del análisis llevado a cabo sobre los 4 sujetos para los tres casos de extracción de características detallados en 2.4.3. Se muestra el porcentaje de TP (verdaderos positivos) así como los falsos positivos por minuto (FP/min). Siempre que la detección se encuentre en un periodo de tiempo inferior al tiempo de reacción medio de la persona se considera correcta la detección.

En la figura 5 aparece mostrado, a modo de ejemplo, para uno de los sujetos, el instante en el que se detecta la presencia de láser. La cruz roja representa la aparición del láser; el círculo azul cian, la detección de parada realizada por la IMU; y la línea vertical azul oscuro los instantes clasificados como clase 1. En todos los casos, la detección es anterior a la parada de la persona.

Como se puede observar, la solución que ofrece un mejor promedio es el caso 3 de extracción de características. Respecto al valor de K, sin embargo, el valor óptimo es variable para cada usuario.

$\mathrm{Al}$ añadir a este último caso la segunda validación indicada en 2.6 vemos cómo se consigue aumentar el porcentaje de TP manteniendo prácticamente constantes la tasa de $\mathrm{FP} / \mathrm{min}$, si bien esto se obtiene para distintos valores de K. Para S1 Y S2 los mejores resultados aparecen considerando $\mathrm{K}=2$ mientras que para $\mathrm{S} 3$ y $\mathrm{S} 4$ ocurre con $\mathrm{K}=4$.
Observando los resultados obtenidos en [9] para el caso del polinomio se tiene la siguiente comparación (Tabla 3). En ella se puede observar una mejora significativa en el porcentaje de verdaderos positivos, pasando de un $30.0 \%$ a un $75.0 \%$, y al mismo tiempo una reducción de los $\mathrm{FP} / \mathrm{min}$ producidos de 6.7 a 4.5 ).

\section{CONCLUSIÓN}

En este trabajo se ha realizado un análisis pseudoonline para la detección de la intención de parar frente a la aparición de un obstáculo por medio de las señales EEG. Continuando con el trabajo realizado en [9] se han estudiado nuevos métodos de extracción de características, proponiendo una selección personalizada de electrodos y ventanas temporales previo a cualquier registro online. Asimismo, se propone añadir al clasificador LDA propuesto en [9] una segunda validación basada en correlación cruzada normalizada inspirada en [6] Con estos cambios se ha conseguido mejorar levemente los resultados obtenidos en otros estudios.

En trabajos futuros, deberá comprobarse el método propuesto con una mayor cantidad de sujetos, así como con pacientes con alguna discapacidad física, para comprobar si se cumplen los mismos patrones que en los sujetos sanos. Por otra parte, se pretende mejorar el método de selección personalizada de electrodos, realizado hasta ahora por simple inspección visual, para automatizar la selección.

El objetivo final será evaluar el funcionamiento del método propuesto con un exoesqueleto real que sea capaz de detenerse cuando el sujeto tenga la intención de ello.

\section{Agradecimientos}

Esta investigación ha sido realizada en el marco del proyecto Walk - Control de exoesqueletos de miembro inferior mediante interfaces cerebromáquina para asistir a personas con problemas de marcha (RTI2018-096677-B-I00), financiado por el Ministerio de Ciencia, Innovación y Universidades (MCIU), la Agencia Estatal de Investigación (AEI) y la Unión Europea a través del Fondo Europeo de Desarrollo Regional (FEDER).

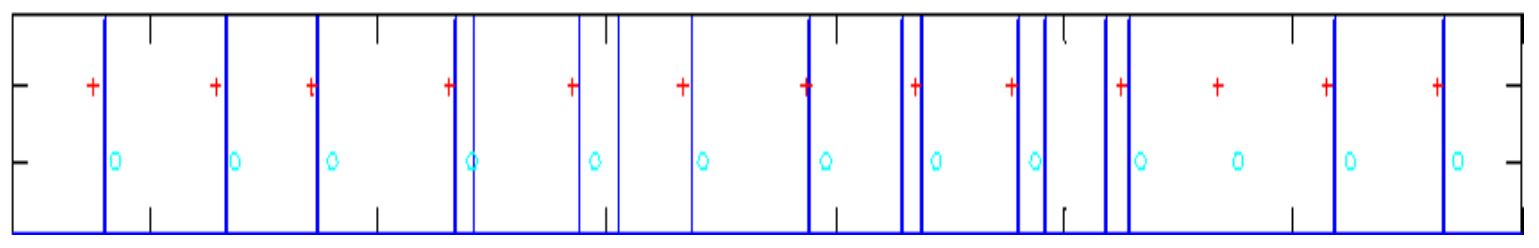

Figura 5. Sujeto $\mathrm{S} 1$ con $\mathrm{K}=2$. 
Tabla 2. Comparativa de resultados pseudo-online para los tres casos de extracción de características

CASO 1

\begin{tabular}{|c|c|c|c|c|c|c|c|c|}
\hline \multirow[t]{2}{*}{ SUJETO } & \multicolumn{4}{|c|}{ TP (\%) } & \multicolumn{4}{|c|}{ FP/MIN } \\
\hline & $\mathrm{K}=1$ & $\mathrm{~K}=2$ & $K=3$ & $\mathrm{~K}=4$ & $\mathrm{~K}=1$ & $K=2$ & $\mathrm{~K}=3$ & $\mathrm{~K}=4$ \\
\hline S1 & 76.9 & 30.8 & 0.0 & 0.0 & 4.9 & 0.0 & 0.0 & 0.0 \\
\hline S2 & 46.2 & 38.5 & 23.1 & 23.1 & 5.4 & 2.7 & 2.1 & 0.0 \\
\hline S3 & 46.2 & 7.7 & 0.0 & 0.0 & 4.2 & 1.1 & 0.0 & 0.0 \\
\hline S4 & 69.2 & 76.9 & 69.2 & 61.5 & 13.9 & 9.6 & 4.8 & 2.7 \\
\hline Media & $51.9 \pm 15.8$ & $38.5 \pm 28.8$ & $23.1 \pm 32.6$ & $2.2 \pm 29.0$ & $7.1 \pm 4.6$ & $3.3 \pm 4.3$ & $1.7 \pm 2.3$ & $0.7 \pm 1.4$ \\
\hline
\end{tabular}

\section{CASO 2}

\begin{tabular}{ccccccccc}
\hline SUJETO & \multicolumn{3}{c}{ TP $(\%)$} & \multicolumn{3}{c}{ FP/MIN } \\
& \multicolumn{1}{c}{$\mathrm{K}=1$} & $\mathrm{~K}=2$ & $\mathrm{~K}=3$ & $\mathrm{~K}=4$ & $\mathrm{~K}=1$ & $\mathrm{~K}=2$ & $\mathrm{~K}=3$ & $\mathrm{~K}=4$ \\
\hline S1 & 76.9 & 76.9 & 69.2 & 61.5 & 4.9 & 2.7 & 2.7 & 1.6 \\
S2 & 69.2 & 69.2 & 69.2 & 69.2 & 5.9 & 4.3 & 3.2 & 2.7 \\
\hline S3 & 23.1 & 2.0 & 1.0 & 0.0 & 3.1 & 2.1 & 1.1 & 0.5 \\
\hline S4 & 76.9 & 76.9 & 61.5 & 61.5 & 8.0 & 4.8 & 3.2 & 1.1 \\
\hline Media & $61.5 \pm 25.9$ & $59.6 \pm 36.3$ & $51.9 \pm 33.0$ & $48.1 \pm 32.2$ & $5.5 \pm 2.0$ & $3.5 \pm 1.3$ & $2.5 \pm 1.0$ & $1.5 \pm 0.9$ \\
\hline
\end{tabular}

\begin{tabular}{ccccccccc}
\hline \multirow{2}{*}{ SUJETO } & \multicolumn{3}{c}{ TP (\%) } & & \multicolumn{3}{c}{ FP/MIN } \\
\cline { 2 - 10 } & $\mathrm{K}=1$ & $\mathrm{~K}=2$ & $\mathrm{~K}=3$ & $\mathrm{~K}=4$ & $\mathrm{~K}=1$ & $\mathrm{~K}=2$ & $\mathrm{~K}=3$ & $\mathrm{~K}=4$ \\
\hline S1 & 76.9 & 76.9 & 69.2 & 69.2 & 6.0 & 2.7 & 2.7 & 2.2 \\
\hline S2 & 69.2 & 69.2 & 69.2 & 61.5 & 5.9 & 4.3 & 2.7 & 2.7 \\
\hline S3 & 15.4 & 15.4 & 7.7 & 7.7 & 2.6 & 1.0 & 0.5 & 0.5 \\
\hline S4 & 84.6 & 84.6 & 61.5 & 53.9 & 8.0 & 4.8 & 2.7 & 1.1 \\
\hline Media & $61.5 \pm 31.4$ & $61.5 \pm 31.4$ & $51.9 \pm 29.7$ & $48.1 \pm 27.6$ & $5.6 \pm 2.2$ & $3.2 \pm 1.7$ & $2.1 \pm 1.1$ & $1.7 \pm 1.0$ \\
\hline
\end{tabular}

\section{CASO 3+VALIDACIÓN EXTRA}

\begin{tabular}{ccccccccc}
\hline SUJETO & \multicolumn{3}{c}{$\mathrm{TP}(\mathbf{\%})$} & \multicolumn{3}{c}{ FP/MIN } \\
\cline { 2 - 10 } & $\mathrm{K}=1$ & $\mathrm{~K}=2$ & $\mathrm{~K}=3$ & $\mathrm{~K}=4$ & $\mathrm{~K}=1$ & $\mathrm{~K}=2$ & $\mathrm{~K}=3$ & $\mathrm{~K}=4$ \\
\hline S1 & 100.0 & 92.3 & 76.9 & 69.2 & 9.2 & 2.7 & 2.7 & 1.6 \\
\hline S2 & 76.9 & 76.9 & 53.9 & 46.2 & 10.7 & 4.8 & 2.7 & 1.6 \\
\hline S3 & 100.0 & 92.3 & 61.5 & 61.5 & 25.2 & 17.8 & 13.0 & 6.8 \\
\hline S4 & 100.0 & 84.6 & 76.9 & 69.2 & 23.0 & 15.0 & 7.5 & 3.8 \\
\hline Media & $94.2 \pm 11.6$ & $86.5 \pm 7.4$ & $67.3 \pm 11.5$ & $61.5 \pm 10.8$ & $17.0 \pm 8.2$ & $10.1 \pm 7.4$ & $6.5 \pm 4.9$ & $3.5 \pm 2.5$
\end{tabular}

Tabla 3. Comparación resultados estudio anterior (tabla izquierda) y actual (tabla derecha)

Estudio anterior [9]

\begin{tabular}{c|c|c|c}
\cline { 2 - 4 } & $\mathrm{K}$ & $\mathrm{TP}(\%)$ & $\mathrm{FP} / \mathrm{min}$ \\
\hline S1 & 4 & 28.6 & 8.0 \\
\hline S2 & 4 & 42.9 & 2.1 \\
\hline S3 & 2 & 42.9 & 8.5 \\
\hline S4 & 2 & 7.1 & 2.7 \\
\hline S5 & 2 & 28.6 & 11.9 \\
\hline Media & & $30.0 \pm 14.6$ & $6.7 \pm 4.2$
\end{tabular}

Estudio actual

\begin{tabular}{c|c|c|c}
\cline { 2 - 4 } & $\mathrm{K}$ & $\mathrm{TP}(\%)$ & $\mathrm{FP} / \mathrm{min}$ \\
\hline S1 & 2 & 92.3 & 2.7 \\
\hline S2 & 2 & 76.9 & 4.8 \\
\hline S3 & 4 & 61.5 & 6.8 \\
\hline S4 & 4 & 69.2 & 3.8 \\
\hline & & $75.0 \pm 13.1$ & $4.5 \pm 1.8$
\end{tabular}




\section{English summary}

\section{NEW METHODS FOR THE DETECTION OF UNESPEXTED OBSTACLES DURING NORMAL GAIT THROUGH EEG SIGNALS}

\begin{abstract}
This paper aims to evaluate new methods for detecting the appearance of an unexpected obstacle during the normal gait from electroencephalographic
\end{abstract}

\section{Referencias}

[1] Abril, J.F., Planelles, D., Costa. Á., Iáñez, E., Azorín, J.M. (2014). "Clasificación de cambio de dirección durante la marcha mediante el uso de señales electroencefalográficas". Actas de las XXXV Jornadas de Automática, Valencia, Spain, pp: 42-48, (3-5 September 2014).

[2] Bellingegni, A. D., Gruppioni, E., Colazzo, G., Davalli, A., Sacchetti, R., Guglielmelli, E., \& Zollo, L. (2017). NLR, MLP, SVM, and LDA: A comparative analysis on EMG data from people with trans-radial amputation. Journal of neuroengineering and rehabilitation, 14(1), 82.

[3] Geffner, D. (2011). El cerebro, organización y función.

[4] Iáñez, E., Costa, A., Úbeda, A., RodriguezUgarte, M., Azorín, J.M. (2016). "A new upgrading model for detecting the reaction to obstacle appearance during walking using EEG". Actas de las XXXVII Jornadas de Automática, pp. 184-189. (7, 8 y 9 de septiembre de 2016, Madrid),

[5] Lebedev, M. A., \& Nicolelis, M. A. (2006). Brain-machine interfaces: past, present and future. TRENDS in Neurosciences, 29(9), 536546.

[6] López, J., Úbeda, A., Iáñez, E., Planelles, D., Azorín, J. M., Gimeno, J., Flores, J.A., Climent, J.M. (2013)." Clasificación de señales de potencial de error a través de una interfaz gráfica con realimentación de fuerzas". Actas de las XXXIV Jornadas de Automática. Terrassa, España, pages: 100-107. 4-6 September

[7] Mallat, S., \& Hwang, W. L. (1992). Singularity detection and processing with wavelets. IEEE transactions on information theory, 38(2), 617643. signals (EEG), reducing the false positive rate obtained in previous studies. This way, in case that an emergency occurs, an exoskeleton for both rehabilitation and assistance to people with a motor disability could be stopped. Our purpose is, therefore, to address the implementation of this exoskeleton in real life, getting greater interaction and involvement of the subject with the system. An improvement in the results has been achieved with respect to a previous study, obtaining $75.0 \%$ success rate and 4.5 false positives per minute ( $\mathrm{FP} / \mathrm{min})$.

Keywords: obstacle, EEG, BMI, exoskeleton

[8] Murie-Fernández, M., Irimia, P., Martínez-Vila, E., Meyer, M. J., \& Teasell, R. (2010). Neurorehabilitation after stroke. Neurología (English Edition), 25(3), 189-196.

[9] Salazar-Varas, R., Costa, Á., Iáñez, E., Úbeda, A., Hortal, E., \& Azorín, J. M. (2015). Analyzing EEG signals to detect unexpected obstacles during walking. Journal of neuroengineering and rehabilitation, 12(1), 101.

[10] Salazar-Varas, R., Costa, A., Úbeda, A., Iáñez, E., \& Azorín, J. M. (2015, April). Changes in brain activity due to the sudden apparition of an obstacle during gait. In 2015 7th International IEEE/EMBS Conference on Neural Engineering (NER) (pp. 110-113). IEEE.

[11] Sanei, S., \& Chambers, J. A. (2007). EEG signal processing. Ed. John Wiley \& Sons, Ltd.

[12] Wada, K., Ono, Y., Kurata, M., Ito, M. I., Minakuchi, M. T., Kono, M., \& Tominaga, T. (2019). Development of a Brain-machine Interface for Stroke Rehabilitation Using Eventrelated Desynchronization and Proprioceptive Feedback. Advanced Engineering, 8, 53-59.

[13] Webb, A. R. (2003). Statistical pattern recognition. John Wiley \& Sons.

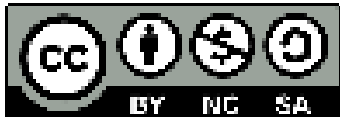

(C) 2019 by the authors. Submitted for possible open access publication under the terms and conditions of the Creative Commons Attribution CC BY-NC-SA 4.0 license (https://creativecommons.org/licenses/bync-sa/4.0/deed.es). 